\title{
JÓVENES CHILENOS Y SUDAMERICANOS: DEMANDAS, ASPIRACIONES Y POLÍTICAS DE JUVENTUD
}

\author{
OSCAR DÁVILA LEÓN*
}

\begin{abstract}
RESUMEN
Sobre la base de un estudio comparado regional sudamericano sobre juventudes, estado de sus demandas y políticas públicas de juventud, se presenta sus dimensiones metodológicas y analíticas para el caso de los jóvenes chilenos, estableciendo las posibles relaciones entre estos tópicos principales. El propósito del estudio se orienta hacia el imaginar caminos donde coincidan los mapas cognitivos de los distintos actores con los itinerarios juveniles, y construir así una política pública adecuada a las vivencias y tránsitos del joven de hoy. Se concluye, que es preciso una política de juventud que responda a las necesidades, intereses y, sobre todo, a los tiempos juveniles; haciendo las distinciones entre los mapas que se elaboran y los itinerarios juveniles, donde la política de juventud debiera ser un tercer elemento y actuar como mediador.
\end{abstract}

PALABRAS CLAVE: JUVENTUD SUDAMERICANA, INTEGRACIÓN REGIONAL, DEMANDAS JUVENILES

* Centro de Estudios Sociales CIDPA. E-Mail: oscar@cidpa.cl. 


\title{
JOVENS CHILENOS E SUL-AMERICANOS: DEMANDAS, ASPIRAÇÕES E POLÍTICAS DE JUVENTUDE
}

\begin{abstract}
RESUMO
Com base num estudo comparado regional sul-americano sobre juventudes, a situação de suas demandas e políticas públicas de juventude, o artigo apresenta suas dimensões metodológicas e analíticas para o caso dos jovens chilenos, estabelecendo as possíveis relações entre estes tópicos principais. O propósito do estudo é pensar caminhos nos quais os mapas cognitivos dos distintos atores coincidam com os itinerários juvenis, construindo assim uma política pública adequada às vivências e trânsitos do jovem de hoje. Conclui-se que é preciso uma política de juventude que responda às necessidades, interesses e, sobretudo, aos tempos juvenis; fazendo as distinções entre os mapas que se elaboram e os itinerários juvenis, onde a política de juventude deveria ser um terceiro elemento e atuar como mediador.
\end{abstract}

\section{PALAVRAS-CHAVE: JUVENTUDE SUL-AMERICANA, INTEGRAÇÃO REGIONAL, DEMANDAS JUVENIS}

\section{CHILEAN YOUTH AND SOUTH AMERICANS: PETITIONS AND POLITICAL ASPIRATIONS OF YOUTH}

\begin{abstract}
On the basis of a comparative study on regional South American youth, their state of petitions and public politics of youth, presents methodological and analytical dimensions to the case of Chilean youth, establishing the possible relationships between these main topics. The purpose of the study was designed to match the imaginative ways in which cognitive maps of different routes with young players and build up a proper public policy to the experiences and transitions of the young of today. We conclude that it must be youth policy that meets the needs, interests and especially time of youth, making distinctions between elaborated maps and youth itineraries, where the youth policy should be a third element and acts as a mediator.
\end{abstract}

KEY WORDS: SOUTH AMERICAN YOUTH, REGIONAL INTEGRATION, YOUTH PETITIONS 
LA INVESTIGACIÓN DENOMINADA «JUVENTUD e integración sudamericana: diálogos para la construcción de la democracia regional», llevada a cabo por un consorcio de instituciones e investigadores de Argentina, Bolivia, Brasil, Chile, Paraguay y Uruguay, durante los años 2007-2009, nos sirve de base para el presente artículo (cf. IBASE, PÓLIS y CIDPA, 2009). ${ }^{1}$

Dicha pesquisa nos permitió adentrarnos desde distintas aproximaciones conceptuales y metodológicas a las realidades juveniles y sus demandas con una perspectiva comparada en dos planos: las comparaciones entre jóvenes de los seis países, y las comparaciones entre aquéllos y adultos de los respectivos países.

De allí que este estudio pueda ser concebido como pionero en su género, pues hasta ese momento no se había desarrollado alguna experiencia de investigación en el área sudamericana sobre juventudes utilizando para ello un conjunto de metodologías y técnicas de carácter cualitativas y cuantitativas que nos aproximaran a los sujetos jóvenes y sus demandas juveniles.

En este texto se exponen los aspectos centrales por los cuales transitó el estudio, sus fases y metodologías utilizadas, y algunos hallazgos, resultados y conclusiones más relevantes.

\section{LOS CAMINOS Y TRAYECTOS}

Desde la primera etapa de la pesquisa vinculada con la juventud e integración sudamericana, se planteó la imagen de la demanda juvenil que se encontraba cautiva o secuestrada por diferentes procesos relacionados con el plano de las políticas de juventud nacionales y los procesos sociopolíticos que acontecen. Pero junto a ello logramos visibilizar y caracterizar un conjunto amplio de expresiones juveniles de carácter grupal, que a través del desarrollo de la pesquisa en sus posteriores etapas, fuimos precisando y complementando, para final-

1 Las instituciones participantes fueron Fundación SES (Argentina), PÓLIS e IBASE (Brasil), Universidad para la Investigación Estratégica en Bolivia (U-PIEB Bolivia), Centro de Estudios Sociales CIDPA (Chile), Base Investigaciones Sociales (BASE-IS Paraguay); y Cotidiano Mujer y Grupo de Estudios Urbanos y Generacionales, Facultad de Ciencias Sociales, Universidad de la República (Uruguay). El financiamiento del estudio, en todas sus etapas, fue del Centro de Investigaciones para el Desarrollo Internacional (IDRC). 
mente y de manera comparativa con otras realidades de espacio del estudio, quedamos con insumos relevantes a la hora de hacer la vinculación entre estas grupalidades juveniles y sus demandas, sean éstas implícitas o explícitas, de acuerdo a su origen, orientación y puesta en escena por parte de los colectivos. Seguramente esas conexiones son el eje más potente de toda la pesquisa, terreno en el cual aún se encuentran líneas de análisis y conclusiones posibles de extraer y descubrir como insumos para todos los actores involucrados en el proceso del ciclo de vida de las políticas públicas dirigidas hacia los jóvenes.

Desde un comienzo, cuando nos ocupamos de estudiar y analizar dos situaciones tipo de agrupamiento, movilización y demanda juvenil, consistente en la sistematización y visibilización de las demandas juveniles identificadas en Chile, a partir de sus formas de organización y los encaminamientos dados. Y también realizar el examen de cómo las diferentes demandas juveniles se jerarquizan e incorporan en el debate público, en las agendas de la sociedad civil y el diseño de políticas públicas de juventud.

De los dos grupos o expresiones juveniles en el estudio original, la experiencia de las agrupaciones y colectivos juveniles que trabajan con el Departamento de Jóvenes de Concepción - de carácter municipal-, toma relevancia pues, si bien la experiencia puede enmarcarse dentro de la categoría de «joven de proyecto», no se agota en ella, permeándose hacia nuevas experiencias profundamente subjetivas, en las que actores, como los media y la industria cultural, toman parte.

El segundo grupo de estudio fue la movilización estudiantil. Luego de la llamada «Revolución Pingüina», que movilizó a más de 800 mil estudiantes secundarios en todo el país por una «educación pública, gratuita y de calidad», nos propusimos pesquisar no sólo la trayectoria y orgánica del movimiento, sino las experiencias de quienes formaban parte de la Coordinadora Tricomunal de Estudiantes Secundarios de la V Región de Valparaíso.

Al entrelazar consideraciones sobre similitudes y diferencias entre ambas experiencias de grupalidad juvenil, encontramos que los jóvenes han desarrollado en el camino estrategias particulares para enfrentar - y también para fugarse - del mundo que viven; en un contexto en el que el ecosistema bidimensional familia/escuela parece agotarse y otros actores toman la delantera en la configuración de sentido para las identidades de estos colectivos juveniles.

El debate con que cerramos esa primera etapa de la pesquisa tuvo una premisa tácita: la generación de conocimiento ha de servir para la 
acción política. Los análisis y experiencias que han resultado de la sistematización y visibilización de la acción juvenil, dan elementos para repensar el lugar que ocupa la política de juventud en el aparato del Estado. Nos convence la idea que la política de juventud debiera legitimarse, más que como mediadora, como el instrumento cartográfico preciso capaz de incluir la complejidad positiva entre itinerarios juveniles y los mapas elaborados por el resto de los actores involucrados. Y la conclusión se dirigía hacia hacer un debate entre el joven de hoy - que se desprende de la experiencia de grupalidad-, su demanda y la política de juventud, para trabajar, finalmente, en la demarcación de un lugar desde donde abordar mejor a los jóvenes del Chile de hoy. Ese tríptico de actores y dimensiones - el joven, su demanda y la política de juventud - también se constituyó en una relación dinámica que nos acompañó durante todo el transcurso de la investigación y que hubo posibilidades y espacios para su profundización.

En un segundo momento, el estudio se ocupó de registrar la presencia, el estado y la orientación de la demanda juvenil hacia el Estado y la política pública que estaban elaborando diferentes agrupaciones juveniles. Para eso se diseñó una estrategia de investigación mediante Grupos Focales, que son un dispositivo técnico de investigación cualitativa en que el equipo de investigación propone una serie de temas para que los participantes del grupo las trabajen de manera individual y luego las discutan colectivamente.

El propósito fue que los Grupos Focales permitieran generar un espacio para que emergieran demandas y se las pudiera entender en el marco de las definiciones que los mismos jóvenes hacen del ser joven. Lo que parecía más pertinente entonces era convocar a agrupaciones que tuvieran un componente «juvenil» importante, y que al menos en principio tuvieran un discurso más o menos articulado que se pudiera traducir en demandas hacia el Estado. En la medida que se registraran las demandas de agrupaciones unidas temáticamente, en la sumatoria se podría registrar la demanda que se hace al Estado desde diferentes ángulos, desde distintas posiciones, y ver, al mismo tiempo, los posibles elementos comunes que pudieran estar articulando esas diferentes demandas.

La pregunta fue cuáles agrupaciones podrían cumplir esos requisitos. Las formas de grupalidad juvenil son múltiples y temáticamente variadas, y por lo mismo era importante que la selección de las temáticas y de las agrupaciones, al menos en principio, permitiera llegar a discursos traducibles en demandas al Estado. Se decidió por cuatro 
tipos de agrupaciones definidas por el campo o el ámbito en que inscribían su acción. En primer lugar, agrupaciones cuya acción se sitúa en el campo de la lucha por la igualdad de derechos de género. En segundo lugar, organizaciones que desarrollan su acción en el ámbito artístico y cultural. En tercer lugar, agrupaciones que de alguna manera estén ligadas al desarrollo de propuestas alternativas de desarrollo y globalización; y en cuarto lugar, jóvenes que pertenecieran a la comunidad de inmigrantes peruanos en nuestro país.

En cada uno de estos grupos la idea no fue convocar ni analizar a una sola agrupación por cada ámbito, sino convocar a distintas agrupaciones unidas por el hecho de desarrollar su trabajo en un ámbito específico. El único grupo en que se manejó un criterio diferente fue en el de los jóvenes inmigrantes. Aquí no importaba tanto la pertenencia a organizaciones previamente existentes, sino simplemente el hecho que el conjunto de convocados compartiera la condición de inmigrante.

Una vez definido el tipo de agrupaciones, se decidió distribuir la realización de los grupos focales de acuerdo a un criterio territorial, que en un país tan extenso como Chile era importante de tener en cuenta. El propósito inicial fue tratar de abarcar la zona norte, la central y la sur, sin por eso perder de vista criterios logísticos que hicieran viable la realización de los grupos en los plazos definidos. Con estos criterios se seleccionó a las ciudades de Iquique en el norte, Valparaíso y Santiago en la zona centro, y Concepción en la zona sur.

En los casos de Santiago y Valparaíso, la asignación de los grupos se hizo considerando la pertinencia temática en el marco de cada realidad local. Para el caso de Santiago se dejó el grupo focal con jóvenes inmigrantes, teniendo en cuenta los niveles que alcanza el fenómeno de la inmigración en la capital. Para el grupo en que participarían organizaciones que trabajan en el ámbito artístico y cultural se optó por la ciudad de Valparaíso, principalmente por el significado que ha adquirido este eje de acción tras la designación de la ciudad como Capital Cultural de Chile y recibir el título honorífico de Patrimonio de la Humanidad por parte de UNESCO. La asignación de los grupos en que participarían organizaciones asociadas a temáticas de equidad de género y al movimiento altermundista se asignaron a las ciudades de Iquique y Concepción, respectivamente. Por último, se realizó una conversación con un grupo de expertos para recoger una mirada externa sobre las condiciones juveniles contemporáneas y discutir acerca de los temas más relevantes producidos por la serie de grupos focales. 
En un tercer momento la pesquisa abordó a nivel nacional los resultados arrojados por la encuesta aplicada a jóvenes y adultos en los seis países contemplados en el estudio. Junto a ello se tuvo en cuenta los pasos dados por las etapas anteriores de la investigación, que ya habían dado ciertas luces y certezas sobre que las juventudes del continente del sur, tienen condiciones, inquietudes e intereses comunes. Que son un sector importante, no hay duda de ello, y la convicción de promover su integración es una tarea que todo ciudadano debería reconocer como fundamental para vivir la democracia. Tanto jóvenes como adultos, pensando una dicotomía, son parte de la sociedad, y ambos actores construyen e imaginan el futuro, unos más anclados en el pasado y en lo que no pudo ser, y otros más esperanzados en lo que se puede intentar cambiar.

Los propósitos de esta etapa, mediante la realización de la encuesta a nivel nacional en cada país participante, fueron profundizar y operacionalizar ciertas nociones sobre juventud, y sobre todo despejar algunas diferencias de opinión entre las generaciones presentes en el estudio. Acercando el concepto de generación a grupos de edad, más un sentido de reconocimiento de grupo social, que comparte un tiempo y espacio. Hacer una comparación entre adultos y jóvenes permite varias cosas, por un lado establecemos una imagen joven, desde el mundo de los adultos y de los jóvenes, respectivamente, y por otro, permite conocer las opiniones sobre diversas dimensiones, donde tienen protagonismo los jóvenes.

El cuarto y último momento de la investigación a nivel nacional, se constituyó en la generación de un Grupo de Diálogo, al cual se convocó a un conjunto de jóvenes que pertenecen a colectivos y movimientos juveniles para conversar y dialogar sobre los caminos posibles de identificar en torno a viabilizar las demandas emergidas desde sus propias voces y percepciones. Se partía por la premisa en el Grupo de Diálogo (y también de la pesquisa), que uno de los elementos recurrentes al momento de analizar las políticas de juventud en Latinoamérica es que fallan al momento de interpretar las demandas de la juventud. Este proyecto de investigación ha intentado ayudar a definir esas demandas.

Los Grupos de Diálogo son una técnica de investigación social que va explorando las diferentes aristas de un tema mediante un conjunto de dinámicas de conversación. El objetivo central de la técnica es entregar herramientas para contextualizar el tema y luego producir diálogos grupales en que los participantes tienen que tomar posición sobre algunas de las alternativas de acción que ofrece un problema. En 
el caso de este estudio en particular, el objetivo del Grupo de Diálogo fue generar una conversación sobre las estrategias y acciones que los jóvenes organizados identifican como las más legítimas y eficaces en la búsqueda de garantías a la condición de «sujetos de derechos» de los jóvenes. Con este ejercicio se esperaba descubrir nudos críticos y recolectar sugerencias concretas que sirvieran tanto para fortalecer diferentes formas de organización de jóvenes que actúan en la sociedad civil como para incidir sobre la construcción de políticas públicas dedicadas a los jóvenes.

En cuanto a la convocatoria, no era fácil convocar voluntariamente a cuarenta organizaciones y además cumplir con los criterios de diversidad temática, territorial y paridad de género. Lo primero fue convocar a algunas de las organizaciones que habían participado en los Grupos Focales que se organizaron en las ciudades de Iquique, Valparaíso, Santiago y Concepción. De esa forma se le daba continuidad a ambas etapas.

Mediante esa combinación de vías de convocatoria se logró reunir finalmente a un total de 40 participantes que pertenecían a 28 organizaciones diferentes. La composición del grupo resultó territorial y temáticamente diversa. Llegaron representantes de dos agrupaciones de Iquique - ambas habían participado en la etapa de los grupos focales-, ocho organizaciones de Concepción, una de Villa Alemana, dos de Quilpué, cinco de Valparaíso, tres de Santiago - dos de ellas con sede en la capital, pero con alcance nacional—, seis de Viña del Mar y una de San Felipe.

El espectro de organizaciones incluyó agrupaciones que defienden los derechos a la diversidad sexual y las prácticas de salud sexual y reproductiva; un conjunto de centros culturales que trabajan a nivel territorial organizando diferentes tipos de actividades; organizaciones que trabajan por la reivindicación de la autonomía cultural y política de los pueblos originarios, fundamentalmente mapuches; dirigentes estudiantiles universitarios; militantes de partidos políticos; miembros de grupos que organizan encuentros para la práctica y difusión de deportes urbanos, específicamente el skate; dirigentes sindicales que integran el Departamento Juvenil de la Central Unitaria de Trabajadores de Chile (CUT); integrantes de organizaciones de mujeres trabajadoras del mundo rural; miembros de agrupaciones ecologistas que trabajan a nivel local e integrantes de radios comunitarias.

Otro aspecto relevante del grupo fue su composición etaria. Hubo desde representantes de diferentes edades, desde dirigentes de estu- 
diantes secundarios que dan lo que podrían ser sus «primeros pasos» en el trabajo dentro de organizaciones, hasta jóvenes actualmente gestores culturales que han tenido una larga trayectoria en el trabajo en distintos tipos de organizaciones (estudiantiles y culturales, fundamentalmente). Esa dispersión etaria hizo del Grupo de Diálogo un espacio de encuentro e intercambio de experiencias entre diferentes generaciones de dirigentes sociales jóvenes.

Estos elementos principales de los cuatro momentos por los cuales transitó la investigación, nos permitió ir realizando un proceso de profundización de los tópicos centrales de la pesquisa, que tanto desde una dimensión cualitativa junto a la cuantitativa, permitió trazar algunos puntos de encuentro y conexión entre las condiciones juveniles, la demanda juvenil y las políticas de juventud.

\section{HALLAZGOS, CONSTATACIONES Y DISCUSIONES}

\section{a) Las relevancias en las situaciones tipo estudiadas}

En la selección inicial de las situaciones tipos a ser estudiadas, se optó por relevar la movilización y movimiento estudiantil secundario, el que tuvo una amplia visibilidad pública durante el año 2006 y posterior, bajo la demanda de transformaciones y cambios en la educación y el sistema educacional chileno. La segunda situación tipo correspondió a las agrupaciones juveniles de la ciudad de Concepción. De ambas experiencias de articulación podemos extraer ciertos elementos de vigencia y recreación sobre las discusiones que vinculan a jóvenes, demandas y políticas hacia la juventud.

De allí que la movilización juvenil en torno a proyectos comunes es una experiencia intensa no sólo en el campo emocional, sino cultural y eminentemente en el campo de lo político. Es en el agruparse, que los jóvenes experimentan sus límites y posibilidades individuales y colectivos.

i) Tanto el movimiento estudiantil como las agrupaciones juveniles en Concepción son acciones juveniles sumamente actuales y que, para efectos de este estudio, constituyen experiencias que pueden sistematizarse en torno a un hito o núcleo principal, también contemporáneo.

En el caso de la movilización de estudiantes secundarios en la V Región, ésta representa la continuación de la llamada «Revolución Pingüina» de 2006, que, sin precedentes, logró instalar la educación 
como problema público poniendo en acción a más de 800 mil alumnos a lo largo del país. En la «Revolución Pingüina» pudimos vislumbrar no sólo la expresión de una demanda hacia la institucionalidad política y el sistema educacional que rige hasta hoy, sino también una interpelación generacional, apoyada fuertemente en un lenguaje de derechos de ciudadanía, hacia quienes deciden esas materias y han operado con una dosis demasiada alta de conformismo con lo heredado.

En Concepción, el contexto que configura el ambiente en el que analizamos las experiencias de grupalización de la juventud es el proceso de integración de nuevas agrupaciones y colectivos juveniles al Departamento de Jóvenes de la municipalidad. Grupos que traen al Departamento nuevas formas de vincularse, nuevas temáticas para agruparse y relaciones que toman fuerza en el campo de la industria cultural. Estas nuevas agrupaciones, llamadas ACTIVA, imprimen en el trabajo de la política de juventud local otra perspectiva sobre cómo abordar las necesidades e intereses juveniles. El hito que instalamos como eje de la situación tipo es el momento en que los ACTIVA proponen que no se de impulso al plan de presupuesto participativo (que implicaba competir entre ellos para conseguir recursos) y, plantean en su lugar, que se financien y fomenten actividades para conocerse entre todos ellos y/o que se repartan los recursos de forma equitativa.

ii) Ambas situaciones tipo configuran maneras particulares de vincularse con el poder público. A primera vista, expresan una demanda muy particular y precisa, pero en su visibilización los jóvenes dejan entrever otras necesidades e intereses. Esto es relevante, pues significa que la expresión de una demanda específica trae consigo rastros de otras preocupaciones en los jóvenes de hoy.

Entre los jóvenes secundarios que se movilizan en la V Región, podemos ver que más allá de la demanda «por una educación pública, gratuita y de calidad» existe una acertada autopercepción de que son capaces de reflexionar sobre la situación que viven y, por lo tanto, demandan un espacio desde donde poder aportar a la construcción del país que queremos, es decir, constituirse en actores sociales relevantes.

Por su parte, en Concepción, la especificidad de la demanda se funda en el trabajo a nivel local que desarrolla el Departamento de Jóvenes de la Municipalidad de Concepción (donde es posible colaborar para pensar y hacer en grande entre jóvenes). La labor que realiza el Departamento consiste principalmente en impulsar y movilizar una diversificación de la relación con la juventud local, desde un nivel instrumental hasta uno intersubjetivo: demanda por acompañamiento, 
apoyo y reconocimiento por parte del poder público y la sociedad civil. En otras palabras, aunque la acción juvenil parte como un proyecto, ésta no se agota en él y, por el contrario, convocan en su accionar a otros actores, fundamentalmente la industria cultural.

En Concepción, la demanda encuentra un estructurado marco de respuestas desde la política pública, gestionado básicamente por medio de fondos concursables puestos a disposición por gran parte de las instituciones del poder político. Sin embargo, una reflexión crítica sobre las agrupaciones juveniles recientes en la comuna, permite trascender la concepción de que los jóvenes de hoy son pasivos (que sólo reciben) y que sus agrupaciones han de ser únicamente pragmáticas o instrumentales.

iii) Al indagar en las agrupaciones o colectivos juveniles nos permitimos comprender, desde la perspectiva de los propios jóvenes, sus vivencias y tránsitos como joven: cómo dialoga con el entorno y por dónde pasan sus críticas y demandas al sistema.

En el caso de la demanda de los jóvenes secundarios, ésta se encuentra dirigida directamente hacia el poder político representado a través del Estado chileno. Existe en el país un amplio consenso en la capacidad que tuvo el movimiento estudiantil para detonar un proceso de incidencia en la Política Educativa de Chile. Esta capacidad detonadora los convirtió, sin lugar a dudas, en actores sociales en el espacio público nacional. Resulta sumamente interesante relevar que, en este caso, la acción juvenil descubrió y recordó al resto de la sociedad el espacio público como un lugar en disputa.

Por el contrario, los jóvenes de Concepción buscan fortalecer su autonomía más que la conformación de una estructura por encima de ellos, procurando disminuir la desconfianza y visibilizando sus acciones. La identidad juvenil de los grupos que participan en el Departamento emplazan su afinidad en lo distinto o disidente bajo el alero de conceptos como contrapoder, autonomía y autogestión; al mismo tiempo que valoran como fin en sí mismo «la existencia cotidiana de los grupos y sus resistencias». Las demandas de los jóvenes que participan en el Departamento se caracterizan por ser de corto plazo. Los jóvenes son en general bastante pragmáticos y se encuentran generando permanentemente nuevas formas de resistencia, de organización, de acción juvenil.

Entre las agrupaciones de Concepción, al momento de establecer vínculos con el poder político, el lenguaje de derechos no es muy común, especialmente en las agrupaciones y colectivos de reciente in- 
corporación, lo que sí sucede en el movimiento estudiantil. En las agrupaciones y colectivos que trabajan con el Departamento se observa poco interés por el carácter temporal o la historicidad de las relaciones entre quienes los componen, lo que pone en jaque la posibilidad de construir una demanda con un contenido profundo, generando más bien una que juega en el campo de la temporalidad privada (demanda acotada, precisa y factible) donde, al parecer, la intensidad tiende a primar por sobre la profundidad.

Las situaciones tipo elegidas no sólo aportan al conocimiento acerca de la orgánica en agrupaciones y colectivos juveniles, sino que abordarlas desde el foco de su demanda, da pie para abordar los discursos que se construyen en la relación con otros actores como el poder público, la sociedad civil y los medios de comunicación. Por un lado, las agrupaciones y colectivos de Concepción mantienen un vínculo estrecho con el poder público local, pues es gracias a él que pueden realizar actividades que van más allá de sus posibilidades individuales. Asimismo, muchos de los grupos ACTIVA han encontrado en la industria cultural la fuente de su identidad, lo que llama a indagar la naturaleza de dicha relación.

Por otro lado, en el movimiento estudiantil, la demanda ha sobrepasado con creces una mera reivindicación sectorial en el campo de la educación; ésta abarca y ha movilizado al conjunto de la sociedad y sus actores, en especial al poder público. Si bien la demanda se levanta como una reivindicación no sólo social sino generacional, la manera en que los distintos actores la han abordado, merece especial atención pues ha de analizarse considerando aspectos sincrónicos y diacrónicos de la histórica social y política del Chile posdictadura, preocupaciones e intereses políticos, así como las agendas mediáticas.

\section{b) El ser joven en los grupos focales: ventajas y desventajas}

Al revisar la discusión que se dio en los distintos grupos sobre el ser joven y sobre sus ventajas y desventajas, se puede extraer un conjunto de tópicos que de alguna manera permiten armar una imagen general sobre el sujeto juvenil. El primer rasgo apunta a un conjunto de cualidades físicas y anímicas que se atribuyen a la juventud. Las referencias a la energía, la vitalidad, el vigor aparecieron en todos los grupos, y se habló de ellas como características inherentes al ser joven. Esas cualidades presentan dos sentidos: por un lado, se refieren a características biológicas que vendrían por las cualidades etarias de esta etapa 
de la vida, pero también se refieren a una disposición, si se quiere, «espiritual» frente a la vida. La energía y la vitalidad no se nombran solamente como una característica del «cuerpo joven»; también constituyen una disposición hacia la acción, y en esa doble acepción definen la primera ventaja que tiene ser joven.

Desde esa disposición hacia la acción se tiende un puente hacia un segundo conjunto de rasgos, que tienen que ver esta vez con la condición social de joven: los grados de libertad y el tiempo. Estos dos componentes del ser joven también aparecieron en todos los grupos, y en todos constituye una de sus principales ventajas. En resumen, la condición de dependencia económica, el hecho que muchos sean estudiantes y la ausencia de responsabilidades familiares disminuyen la carga, amplían los grados de libertad y el tiempo disponible, todos elementos que son parte de las ventajas que tiene ser joven.

El tercer rasgo común que define este perfil del ser joven se asocia a una serie de cualidades espirituales - idealismo, crítica, rebeldía, propuesta - que tienen que ver de alguna manera con la sensación de tener todavía un futuro que está abierto, que (todavía) no impone exigencias, que no ha llegado a «someter», que es lo que le pasaría al ser adulto. Todas las ventajas que logran identificar tienen un sentido relativo: no son ventajas en sí, sino respecto a la condición del ser adulto.

Lo interesante es que en este punto se pueden percibir matices de diferencia asociados al perfil de los participantes de cada grupo. Los matices tienen que ver con los sentidos de la acción que mueve la energía juvenil y que facilita el disponer de menos carga de responsabilidad y más tiempo. De manera esquemática, se pueden definir dos grupos dependiendo del sentido que le otorgan al uso de esa energía, de esa libertad y de ese tiempo. El primero lo forman los tres grupos en que participaron agrupaciones de jóvenes chilenos (pro-equidad de género; gestores culturales y altermundistas). El factor que los agrupa es que la energía, el tiempo y las ideas del ser joven confluyen en una acción orientada a la transformación de la realidad, sea cultural, social, económica o estructural. La energía es para la lucha; el tiempo es para organizarse, para actuar y crear ideas; las ideas son para elaborar crítica y proponer transformaciones. En ese sentido, ser joven de alguna manera adquiere un carácter «ideológico», que si bien no debiera llevar a la naturalización esencialista del «joven revolucionario», porque también hay adultos que pueden serlo, al menos sí justificaría una relación entre juventud y transformación, principalmente porque tienen las condicio- 
nes anímicas —energía, ideas - y sociales — ausencia de cargas, tiempo- para formar agrupaciones y generar acción.

El segundo grupo corresponde al de los jóvenes inmigrantes. Lo que caracteriza a este grupo es que, si por un lado comparten buena parte de las características y atributos que definen al ser joven - energía, sueños, metas-, el sentido que le dan a la acción no desemboca directamente en procesos de transformación social, sino en un cambio en sus propias condiciones de vida. La energía, la vitalidad, la capacidad, la resistencia propias del ser joven se traducen en capacidades para trabajar; la libertad que otorga la ausencia de cargas de responsabilidad facilita la decisión de emigrar y buscar mejores oportunidades.

En el relato de los jóvenes inmigrantes, la ventaja de ser joven se presenta como una alternativa para superarse o ampliar sus horizontes mediante el trabajo y la educación, como un período clave para mejorar su calidad de vida, salir adelante, ser alguien en la vida. Es decir, la juventud sí tiene un sentido de transformación de la realidad, pero esa transformación pasa primero por un cambio en las condiciones de vida del inmigrante y no necesariamente por una transformación sistémica, aunque en la práctica esos cambios en la situaciones personales y colectivas de los inmigrantes a la larga también se puedan llegar a traducir en cambios estructurales y culturales más profundos.

En lo que se refiere a las desventajas, por su parte, las referencias se pueden resumir en dos planos: el de las desventajas estructurales y el de las desventajas específicas asociadas a la condición juvenil propiamente tal. Al primer grupo corresponde el conjunto de desventajas asociadas a la inequidad. Los problemas de acceso y financiamiento de estudios superiores, las desventajas en el acceso a las nuevas tecnologías, las diferencias de salario que afectan a los jóvenes cuando trabajan, son algunos de los ejemplos que más grafican este nivel de desventajas. El punto es que no son desventajas que afecten igualmente a toda la juventud; por el contrario, este tipo de desventajas en el fondo aparece cuando se hace referencia a las diferencias de clase entre los mismos jóvenes. Eso de alguna manera ayuda a nivelar las diferencias que pudiera haber entre las desventajas que plantean los jóvenes chilenos y los inmigrantes, pero también impide definirlas como una desventaja propia y exclusiva de la población joven. Al ser desventajas de clase, su efecto se hace transversal, afectan al adulto y al joven, aunque en este último caso su efecto se potencia, porque son ellos quienes «todavía se están haciendo», como se dijo en un grupo. 
Las desventajas en el segundo plano se refieren a una serie de situaciones que parecieran ser patrimonio exclusivo de la población joven. La clave en este punto está en la relación jóvenes/adultos, en especial en la relación jóvenes/instituciones, y los conceptos que quizá las resumen de mejor manera son descrédito, la falta de espacios y discriminación. El descrédito se refiere a la falta de fe que en general deposita el mundo adulto en las iniciativas de los jóvenes. Esta sensación de descrédito fue patente en todos los grupos, aunque especialmente en los grupos que convocaron a jóvenes chilenos. El descrédito se vierte sobre sus iniciativas porque el mundo institucional-adulto por un lado duda de sus propuestas, pero al mismo tiempo les teme por los «subversivo» que pudieran ser. Lo mismo ocurre con la falta de espacios, que también fue un término nombrado principalmente en los grupos con jóvenes chilenos más que en el de jóvenes inmigrantes, quizá porque la comunidad inmigrante se ha ido apropiando de algunos espacios públicos, o por una ausencia real de instancias de ocupación y participación por parte de agrupaciones juveniles que se mueven en ámbitos que efectivamente requieren de espacios físicos y simbólicos: los artistas, teatros; los de medios de comunicación, espacio radial.

La discriminación, por su parte, aparece como un producto del descrédito que afecta a las iniciativas juveniles, afecta a las mujeres jóvenes cuando los marcos legales les coartan sus posibilidades de decidir sobre su cuerpo en períodos de fertilidad, se expresa también en el mundo laboral al momento de intentar acceder a un trabajo - juicio a la inexperiencia-, pero se acentúa todavía más en el caso de los inmigrantes, que a su condición de jóvenes y pobres, se les agrega una condición de inmigrante que deja más expuesto a discriminaciones salariales, abusos laborales y policiales, y discriminaciones raciales.

\section{c) Demandas desde los jóvenes en los grupos focales}

Al momento de resumir las demandas aparecen dos niveles. En primer lugar, se plantea una serie de demandas generales que tienen que ver con los problemas de equidad y que apuntan al Estado como sistema: el cambio constitucional, las demandas por educación de calidad y gratuita, las demandas al sistema de salud, las demandas laborales y las demandas por participación social. En el segundo plano, por su parte, aparece un conjunto de demandas más específicas, que de alguna manera se vinculan a la particularidad de cada grupo focal. 
En el primer nivel se trata de demandas transversales que apuntan a mayores y mejores garantías sociales. Éstas son las que aparecen con más frecuencia en los relatos. Por una parte nos encontramos con peticiones de mejora que radican en cambios estructurales y legales del sistema - cambio constitucional y del sistema neoliberal - y por otra con situaciones de discriminación que afecta a los jóvenes especialmente en su relación con el mundo adulto e institucional. La discusión en todos los grupos pasa por estos aspectos y sobre la base de estas demandas es donde se estructura un pensamiento contestatario de los jóvenes hacia el sistema económico, político y social, aunque fue en los grupos de jóvenes chilenos que se expresó de manera más radical la demanda por cambios estructurales como única forma de generar cambios reales en la sociedad.

En el segundo plano, las demandas se van haciendo un poco más específicas dependiendo de los intereses comunes de cada grupo. En el caso de las jóvenes del grupo pro-equidad de género, las demandas específicas se enfocan en el respeto y autonomía del cuerpo y libertad de decisión. Para los gestores culturales, sus demandas específicas se relacionan directamente con la carencia de espacios físicos y simbólicos para el desarrollo de la cultura y las artes, o para el desarrollo de medios de información y comunicación comunitarios. En el grupo de altermundistas, las demandas más particulares también se dirigen hacia la generación de espacios para el desarrollo de actividades. En el grupo de los inmigrantes sus demandas contienen aspectos más enfocados al respeto de derechos, no discriminación y garantías sociales.

En el grupo de inmigrantes, las exigencias o demandas no radican principalmente en transformaciones sociales y de cambio estructural drástico, sino más bien en el reconocimiento de ellos como seres con derechos igualitarios. Como se señaló, el contexto es sin duda un factor relevante a la hora de analizar las demandas presentadas por los jóvenes. Para los inmigrantes, los motivos principales de trasladarse fuera de país fueron mejorar su calidad de vida y ampliar sus expectativas y horizontes, mediante el trabajo. Por tanto, las demandas presentadas se refieren a ese ámbito en particular que los atañe directa y particularmente. Tal es el caso de la educación para los hijos de inmigrantes y el acceso a la salud.

Cuando en los grupos comparan sus demandas con las identificadas por los países sudamericanos en la primera parte de este estudio, advierten bastantes similitudes. De este modo, aluden a que al existir demandas comunes existe una posibilidad de cambio regional, porque 
«la cosa está funcionando dentro de Latinoamérica creo que es posible que las cosas resulten, pero claramente que no enseguida, pero me parece como viable en que tanta gente esté pensando en que hay que hacer todos esos cambios que están mencionados aquí en la hojita y que estamos trabajando en eso, sino, no estaríamos aquí».

\section{d) La mirada cuantitativa de jóvenes y adultos sobre juventudes}

A partir del análisis de la encuesta de juventud sudamericana, se presentan algunas dimensiones sintéticas sobre ciertas situaciones relacionadas con los jóvenes en estudio, intentando dar cuenta de las semejanzas y diferencias entre las miradas y percepciones de adultos y jóvenes chilenos.

En un primer momento en el análisis de la encuesta, se dedicó a destacar una de las principales características del estudio, que permitió realizar una comparación de opiniones y percepciones entre los rangos de edad. Por un lado, tenemos cómo se definen los jóvenes, y por otro, cómo son definidos por los adultos. Lo que se trató de conseguir con esto, es que los datos confirmaran o rechazaran ciertas ideas o condiciones que son asociadas a la edad, pero que también responden a imágenes, como por ejemplo el sentirse joven, o pensar jóvenes a los otros mediante ciertos atributos que ellos no poseen, partiendo de algunas características. Donde muchas veces juega más la juvenilización de ciertos atributos, que las verdaderas cualidades que los jóvenes ven sobre sí mismos.

Como primer antecedente es que los encuestados al posicionarse respecto a las categorías: adolescente, joven, adulto, persona de edad o adulto-joven, sus respuestas se corresponden a la edad, es decir, los primeros tramos de edad se identifican como jóvenes, con fuerza hasta los 29 años. Sin embargo, un 49,8\% del total de los entrevistados se describe adulto, y de ellos un tramo de jóvenes se identifica con cierta importancia en tal categoría, pues a esa edad suponemos una carga de responsabilidades que son asociadas al mundo de los adultos. De alguna forma lo anterior refuerza ciertas nociones sobre la juventud, la cual limita el paso de una condición a otra, una vez tomadas ciertas responsabilidades y no en cuanto a la edad.

El análisis del concepto más cercano al evocar la palabra juventud está orientado por los resultados de la etapa cualitativa de esta investigación. Los grupos focales en esa oportunidad, para el caso de Chile, mostraron la necesidad de entender el ser joven como un con- 
cepto relacional, porque la conversación giro en torno a «cualidades 'esencialistas' que construyen un sujeto en cuatro dimensiones: físicobiológicas (cuerpo joven); anímicas (energía, vitalidad); condiciones sociales (tiempo, libertad); ideológicas (crítica, sueños)». Además, sólo toma sentido hablar de joven en contraste o diferencia a las cualidades que tendrían los adultos, por lo tanto vemos que la construcción de los sujetos jóvenes, en general, ser enmarca en una serie de libertades, oportunidades y expectativas que la misma sociedad abre o cierra según la condición etaria.

\section{TABLA 1: PRIMERA PALABRA CON LO QUE ASOCIA JUVENTUD (ANÁLISIS DE RESPUESTA MÚLTIPLE)}

\begin{tabular}{|l|c|c|}
\hline Juventud & Frecuencia & Porcentaje \\
\hline Alegría & 179 & 9 \\
\hline Fiestas & 141 & 7 \\
\hline Libertad/Independencia & 96 & 5 \\
\hline No sabe & 77 & 4 \\
\hline Diversión & 58 & 3 \\
\hline Futuro & 53 & 3 \\
\hline Falta de responsabilidad/Irresponsabilidad & 50 & 2 \\
\hline Vida & 48 & 2 \\
\hline Energía & 47 & 2 \\
\hline Desorden & 43 & 2 \\
\hline Vivir bien & 41 & 2 \\
\hline Joven/Adolescente & 37 & 2 \\
\hline
\end{tabular}

Fuente: Encuesta Juventudes Sudamericanas, 2009.

Entre el grupo de jóvenes sobresalen aquellas que aluden a condiciones sociales: libertad/independencia, diversión, desorden y vivir bien; además se presentan del tipo anímica: alegría, energía, esfuerzo; y en menor presencia las físico-biológicas y las de tipo ideológico. Los adultos en cambio, si bien comparten gran número de calificativos para la juventud, ellos agregan características negativas como la irresponsabilidad, e incluso caracterizan en mayor medida a la juventud como una etapa en donde se asumen responsabilidades, una especie de llamando a los más jóvenes al cómo asumir esta etapa. Misma categoría que se repite cuando se pregunta por lo que debiera ser el período de la juventud, son los adultos los que piensan que asumir responsabilidades y el prepararse para el futuro, deberían ser el centro de este período. 
GRÁFICO 1: LA JUVENTUD DEBIERA SER... SEGÚN GRUPOS DE EDAD

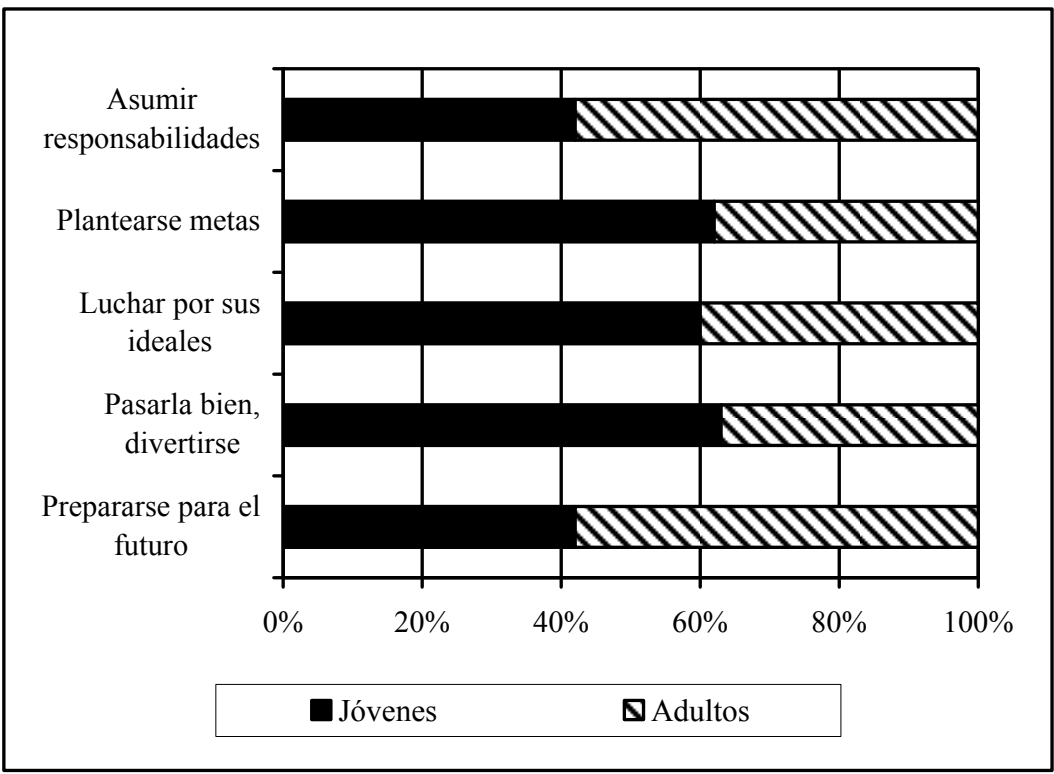

Fuente: Encuesta Juventudes Sudamericanas, 2009.

Se deja ver la diferencia en cómo definimos un concepto, que depende de alguna manera del posicionamiento, ya que los adultos ven a los jóvenes como una experiencia externa o la que viven con sus hijos, pero al mismo tiempo se entremezcla con su propia experiencia de juventud. Ambas definiciones guardan detrás la tarea que han asumido los adultos, la cual es preparar y corregir a las nuevas generaciones. En el lado opuesto están los jóvenes, donde el período de juventud debiera ser para pasarla bien y divertirse, junto a plantearse metas y luchar por lo ideales. Tres esferas que configuran un período, donde se debe conjugar lo que se espera de ellos, con lo que ellos quieren, ya no en el plano de lo ideal.

Hasta el momento la intención de contrastar los datos, ha servido para posicionar las opiniones sobre la etapa. Pensándola de esa manera, se puede caer en el error de sesgarlo a una definición de moratoria o preparación, como la piensan los adultos, y también los mismos jóvenes. Esto hace perder el valor que tiene en sí misma la juventud. En los gráficos siguientes, las opiniones sobre las características se definen en un plano de contraste de ambos grupos de edad, es decir, si 
ellos se consideran más o menos que los adultos, y a la inversa, bajo una lista de atributos seleccionados.

El análisis por grupo edad, nos muestra que son los mismos jóvenes que se piensan a sí mismos ampliamente menos responsables, confirmando lo que los adultos piensan de ellos, sucediendo lo mismo con el atributo trabajadores. Otros que destacan de los gráficos, es que los mismos jóvenes piensan a la juventud, más violenta y peligrosa que los adultos. Ciertamente esas imágenes son más frecuentes en el grupo de mayor edad. Las demás, se comportan sin mayores diferencias entre los grupos.

\section{GRÁFICO 2: COMPARACIÓN DE ATRIBUTOS DE ADULTOS Y JÓVENES}

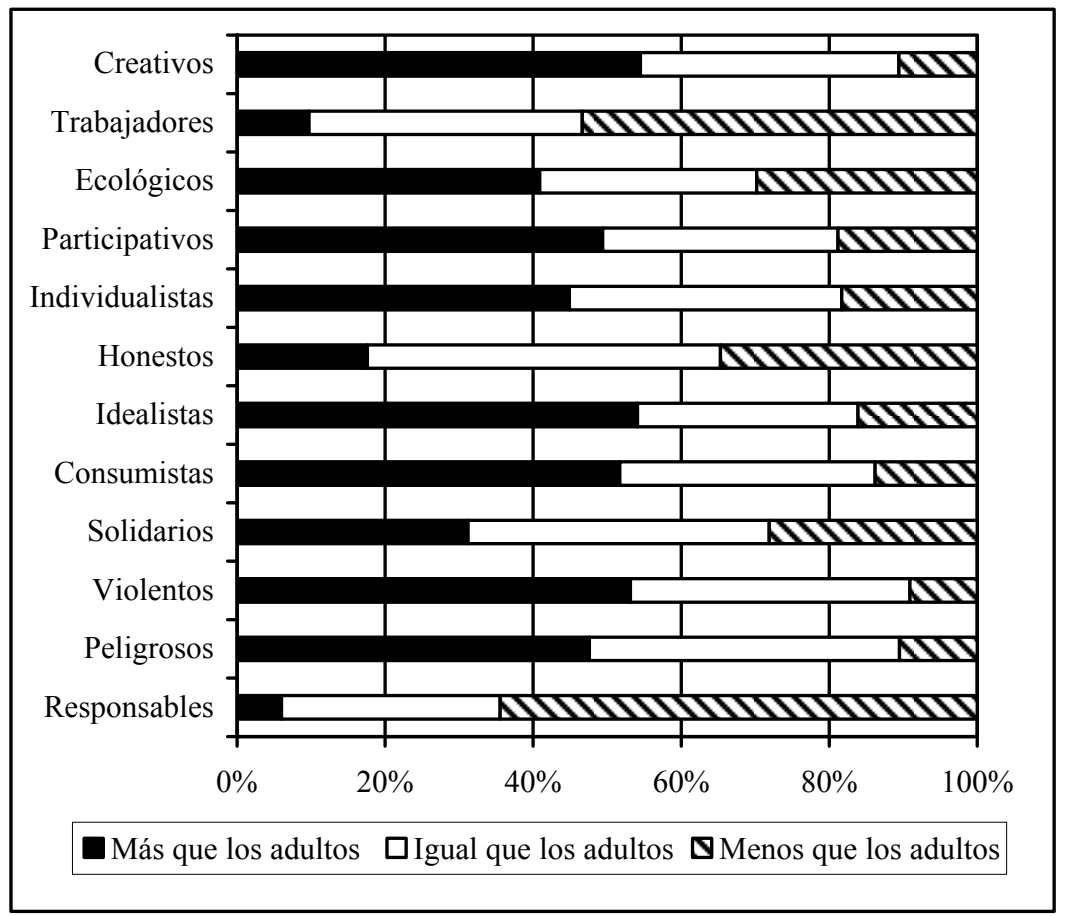

Fuente: Encuesta Juventudes Sudamericanas, 2009.

El principal obstáculo para los jóvenes, según los entrevistados, es la educación de baja calidad que recibe más del $40 \%$ de la población de estudiantes. Este tema se ha levantado como bandera de lucha de amplios sectores, no sólo estudiantes, que ve la segmentación en el plano 
de la educación como fiel reflejo de lo que somos como sociedad. Además, esto no es sólo un problema hoy, muchos de los obstáculos hoy, educación, trabajo y pobreza, son los desafíos con los cuales las poblaciones jóvenes deberán enfrentarse en el futuro. Como lo plantean los encuestados, el trabajo acompañado de un sueldo digno, estudiar y tener un diploma es una preocupación, que no está asegurada, por lo menos hoy, para todos jóvenes.

En el nivel de las acciones hacia la juventud por parte del Estado, la evaluación que hacen los entrevistas es muy baja, no reconociendo ese plano de planes, programas y acciones que la institucionalidad realiza oreintado hacia el mundo juvenil. El reconocimiento por parte de la población en un 59,8\% de los encuestados dice que se conocen las necesidades, pero no se hace nada, siendo los jóvenes los más críticos.

TABLA 2: PERCEPCIÓN DE LAS ACCIONES PARA LA JUVENTUD POR PARTE DEL GOBIERNO

\begin{tabular}{|l|c|c|c|}
\hline Acciones para la juventud & Joven & Adulto & Total \\
\hline $\begin{array}{l}\text { No conoce ni se preocupa } \\
\text { de las necesidades de los jóvenes }\end{array}$ & 20,3 & 15,3 & 16,7 \\
\hline $\begin{array}{l}\text { Conoce las necesidades de los jóvenes, } \\
\text { pero no hace actúa es esta área }\end{array}$ & 61,8 & 59,0 & 59,8 \\
\hline $\begin{array}{l}\text { Apoya y promueve programas y acciones } \\
\text { dirigidas para a juventud }\end{array}$ & 12,9 & 17,8 & 16,4 \\
\hline Ninguna de ellas & 1,1 & 0,8 & 0,9 \\
\hline No sabe & 3,4 & 5,5 & 5,0 \\
\hline Sin respuesta & 0,5 & 1,7 & 1,4 \\
\hline Total & $\mathbf{1 0 0 , 0}$ & $\mathbf{1 0 0 , 0}$ & $\mathbf{1 0 0 , 0}$ \\
\hline
\end{tabular}

Fuente: Encuesta Juventudes Sudamericanas, 2009.

En tabla siguiente se muestran las acciones más o menos importantes para los encuestados. Las que obtienen mayor número de frecuencia son aquellas más visibles, que cuentan con mayor cobertura e inciden en ámbitos cotidianos de la vida de las personas. Sin embargo, hay que destacar que entre las más importantes se encuentran acciones ligadas a problemáticas juveniles y beneficios, es decir, ha permanecido visible en la acción del Estado esa orientación. 
TABLA 3: ACCIONES PARA LA JUVENTUD MÁS IMPORTANTES (RESPUESTAS MÚLTIPLES)

\begin{tabular}{|l|c|c|}
\hline Acciones & Frecuencia & Porcentaje \\
\hline $\begin{array}{l}\text { Programas de apoyo para volver a la escuela } \\
\text { o evitar la deserción }\end{array}$ & 955 & 16,8 \\
\hline $\begin{array}{l}\text { Transporte totalmente gratuito o con } \\
\text { descuento para jóvenes } \\
\text { (pase libre, boleto escolar) }\end{array}$ & 764 & 13,4 \\
\hline $\begin{array}{l}\text { Políticas de salud para jóvenes (prevención } \\
\text { o atención de casos de SIDA, embarazo no } \\
\text { deseado) }\end{array}$ & 743 & 13,1 \\
\hline $\begin{array}{l}\text { Programas de apoyo para inclusión laboral } \\
\text { de jóvenes }\end{array}$ & 736 & 13,0 \\
\hline $\begin{array}{l}\text { Políticas de tratamiento para jóvenes } \\
\text { involucrados con el consumo de drogas } \\
\text { ilícitas }\end{array}$ & 584 & 10,3 \\
\hline $\begin{array}{l}\text { Acciones de cultura, deporte y esparcimiento } \\
\text { para jóvenes en plazas y parques de la ciudad }\end{array}$ & 569 & 10,0 \\
\hline $\begin{array}{l}\text { Apoyo para la inclusión social de jóvenes } \\
\text { con antecedentes delictuales }\end{array}$ & 549 & 9,7 \\
\hline $\begin{array}{l}\text { Acciones y programas para incluir jóvenes, } \\
\text { mujeres, indígenas, afrodescendientes } \\
\text { y personas con alguna discapacidad }\end{array}$ & 417 & 7,3 \\
\hline $\begin{array}{l}\text { Programas de acceso a la tierra } \\
\text { para agricultores jóvenes }\end{array}$ & 358 & 6,3 \\
\hline Ninguna & $\mathbf{5 . 6 8 4}$ & $\mathbf{1 0 0 , 0}$ \\
\hline Total & & \\
\hline
\end{tabular}

Fuente: Encuesta Juventudes Sudamericanas, 2009.

Se reconoce manifiestamente que el paso del programa o acción del Estado a la política de juventud, está escasamente desarrollado. Pues no hay un objetivo detrás que involucre a los jóvenes en tales acciones, la desagregación por sectores ha sido una de las tareas más difíciles de resolver.

\section{e) Demandas, caminos y discursos desde el grupo de diálogo}

La metodología de trabajo para el Grupo de Diálogo considera una serie de momentos que van hilando la discusión. Un primer momento de información. El propósito fue que los participantes entendieran el 
proceso en el que se enmarcaba el Grupo de Diálogo. Al momento de la inscripción se les obsequió un ejemplar de los textos producidos en las dos etapas anteriores de la investigación (situaciones tipo y grupos focales) y luego se hizo una presentación general del proyecto.

Un primer trabajo grupal fue una conversación sobre el listado de siete demandas que habían sido definidas en una etapa anterior del estudio. Para este ejercicio se conformaron ocho grupos. Siete discutirían una de las siete demandas cada uno, y el octavo debía agregar una nueva demanda que no estuviera en la lista, que en definitiva se decidió por el reconocimiento a los derechos de los pueblos originarios. $\mathrm{La}$ pregunta que tendría que resolver cada grupo era si estas demandas constituyen o no un derecho y en qué medida se encuentran garantizados en Chile.

La discusión sobre la condición de derecho juvenil de las demandas fue bastante similar y se centró básicamente en dos interrogantes: si las demandas son derechos exclusivamente juveniles, y en las condiciones para asumir que los derechos están plenamente garantizados por el Estado. Sobre el primer punto, la conclusión general es que en todos los casos, más que de demandas y derechos de los jóvenes, se trata de demandas sociales generales extensibles a todos los grupos de población. Demandas como el transporte, la salud, la educación, el trabajo, el acceso a la cultura, la garantía de un ambiente limpio, son demandas por derechos sociales que están lejos de restringirse solamente a los jóvenes. Que hayan sido dichas por jóvenes no las hace demandas y derechos de la juventud.

Sobre el segundo punto, la conclusión a la que llegaron todos los grupos fue que si bien en algunos casos se reconocen avances, con políticas de acceso y legislaciones específicas, ninguno de estos derechos está plenamente garantizado en Chile. En derechos como la salud y la educación, por ejemplo, si bien el Estado les da ese estatuto, lo hace sólo nominalmente, no en la práctica. El factor que marca esa distancia pasa por las desigualdades estructurales. No se puede asumir que el trabajo se reconoce como un derecho si en la práctica el trabajo digno es realidad de pocos. Cuando se ponen sobre la mesa las desigualdades que produce y reproduce, el trabajo deja de ser derecho garantizado.

Lo mismo ocurre con la educación: la legislación chilena la reconoce como un derecho, pero en la práctica las desigualdades educacionales y la ausencia de una política pública que las reduzca impiden asumirlo como un derecho garantizado. El acceso no basta. La garantía exige la igualación de condiciones en acceso y ejercicio pleno. 
El segundo momento de discusión temática fue sobre los caminos o las estrategias a seguir para garantizar los derechos de los jóvenes. Para introducir elementos que armaran el contexto para este segundo momento se presentó parte de los datos que produjo la encuesta que forma parte de este mismo proyecto de investigación. La idea fue que los participantes del encuentro pudieran comparar el comportamiento de la población chilena y el de la población de los otros cinco países en una serie de opiniones (aborto, pena de muerte, legalización de la marihuana, entre otros) y comportamientos (participación en organizaciones, etc.) que podían servir de insumos para la discusión.

Posterior a eso se expuso el contenido de la discusión, se explicó su sentido, se describieron los caminos y distribuyeron los grupos. La composición de cada grupo había sido preestablecida por el equipo coordinador. El criterio fue darles diversidad temática y garantizar paridad entre sexos.

Entre ambos momentos se aplicó la Ficha Inicial dispuesta en la pauta de trabajo. Con este instrumento se pudo sondear la adhesión que generaba cada uno de los tres caminos antes de la conversación grupal. Los datos de este instrumento arrojaron que, en principio, el camino con más adhesión fue el que propone que las organizaciones juveniles establezcan alianzas con organizaciones con otro perfil y se integren en luchas sociales generales (camino 2). Este fue también el camino en que hubo menos diferencia de opinión. Lo contrario ocurrió con la propuesta de fortalecer solamente las organizaciones juveniles (camino 1). Fue la que menos adhesión generó y en la que las opiniones fueron más discordantes.

Después del diálogo grupal, la mayor parte de los participantes mantuvo su opinión inicial. El orden de importancia siguió siendo el mismo, con la alternativa de vincular las demandas juveniles con las demandas generales de la sociedad como la opción con más fuerza, y con la alternativa de espacios estrictamente juveniles como la que menos sentido hace. Un dato interesante es que, al observar el conjunto de las opiniones en el primer y segundo momento, los tres caminos presentan bajas en su valoración. La explicación es que uno de los resultados del diálogo pareciera ser la construcción de una salida intermedia que suaviza las respuestas extremas de adhesión y rechazo respecto a cada uno de los tres caminos.

El espacio que contemplaba la Ficha Final para fundamentar la segunda respuesta es una primera entrada que permite captar las razones tras las tendencias. Sobre el camino 1, los argumentos de los participantes señalan que el fortalecimiento de las organizaciones juveniles 
es un paso fundamental que hace posible el despliegue del «inevitable impacto social» que se atribuye a la juventud cuando se organiza, pero que para algunos pierde potencia cuando su acción se cierra en demanda estrictamente juveniles y las organizaciones se forman por criterios de edad o de género y no afinidad temática. En ese sentido, la organización juvenil es importante, pero como «primer paso para algo mucho más grande».

Sobre el camino 2, las observaciones valoran positivamente el intercambio de experiencias con adultos, pero sin que eso implique subordinación, pérdida de las especificidades juveniles ni cooptación por parte de los adultos.

Y sobre el camino 3, lo que se dice es que el vínculo con instituciones gubernamentales es importante para avanzar hacia la garantía de los derechos de los jóvenes, "siempre y cuando sea como mutuo fortalecimiento y no en una lógica asistencial», se incluya a la diversidad de visiones y experiencias, con mecanismos eficaces de participación y diálogo, la presencia de todas las organizaciones y que se aboquen a las demandas concretas y genuinas de las organizaciones.

Por eso finalmente los movimientos hacia posturas intermedias en la escala de adhesión para cada camino propuesto, que se resume en una observación general que formula en su ficha uno de los participantes: «mezcla de las tres: fortalecer la organización joven, fortalecer los vínculos y trabajar con instituciones».

\section{TENSIONES, DESAFÍOS Y PREGUNTAS ABIERTAS}

Los jóvenes chilenos y las condiciones - objetivas y subjetivascomo viven actualmente su juventud están cambiando de manera acelerada. Para nadie es sorpresa esa premisa, no obstante las valoraciones y significaciones que se asocian a aquélla. Ahí es donde aparecen interpretaciones encontradas e intencionadas. Este ámbito de análisis se constituyó en un primer eje que cruzó toda la investigación y que a través de sus abordamientos cualitativos y cuantitativos se intentó profundizar y clarificar esta noción de cambio acelerado.

Los otros dos ejes centrales de la pesquisa se vincularon directamente con el anterior y los propósitos fundamentales de este trabajo, estando constituidos por el campo que cubre la participación, sociabilidad, organización y grupalidades juveniles, y la presencia, estado, precisión, orientación y estrategias de acción de la demanda juvenil en cada país. El tercer eje de análisis abarcó el campo de las políticas 
públicas de juventud, las cuales en variadas ocasiones sólo se expresan en un conjunto desarticulado y disperso de planes, programas, proyectos y acciones dirigidas a los jóvenes desde la institucionalidad, no necesariamente juvenil, sino que sectorial.

Por esos tres ejes centrales, temáticos y discursivos, transitó el proceso de pesquisa y análisis, con el propósito de generar conocimientos e insumos que colaboren con el ciclo de vida de las políticas de juventud, y también en el impacto de los colectivos y organizaciones juveniles en cuanto a precisar y potenciar sus demandas y procesos internos de expresividad y movilización juvenil.

\section{a) EI ser joven en el Chile actual}

La construcción institucional de lo que significa ser joven ha marcado profundamente las políticas sociales vinculadas a la juventud. El amplio panorama de miradas que sobre juventud tienen las instituciones de gobierno, puede recogerse en dos discursos o miradas.

Una primera, que concibe al joven como gestor de su proyecto de vida. Éste es un joven que acude a los servicios del Estado a pedir información para capacitarse y conseguir los recursos disponibles para su desarrollo; en tanto sujeto político, es un joven a quien el mundo adulto le propone repetir las mismas acciones del pasado, en otras palabras: que organice y protagonice - cabalmente - demandas históricas, como lo son la educación y el trabajo. A este joven, estratega de su propio desarrollo, están dirigidas las políticas del ramo, un joven emprendedor que ha de saber «manejar las tecnologías y entender el mundo globalizado», porque sólo de esa manera podrá integrarse de forma eficaz a una sociedad competitiva.

La segunda mirada es menos recurrente, y consiste en abordar al joven de hoy desde su subjetividad. Indagar, por ejemplo, en qué quieren, qué piensan, recoger las nuevas demandas en su cotidianeidad, contribuye a reconocer lo que significa ser joven hoy.

Abordar a los jóvenes de hoy desde la segunda mirada es algo muy complejo; muchas de las actividades que realizan los jóvenes están «lejos de la comprensión adulta» lo que hace aún más difuso el panorama. En las agrupaciones y colectivos de jóvenes se discuten temas éticos, de valores y formas de relacionarse que los adultos no son capaces de traducir, principalmente porque la «socialización que los jóvenes desarrollan es al margen de los adultos $\mathrm{y}$, por consiguiente, también de la institucionalidad pública». Las relaciones de los jó- 
venes con el mundo adulto son, en general, tensionadas, selectivas y aparentemente muy instrumentales.

Un acercamiento desde la subjetividad juvenil al sentido que tiene vincularse con su entorno, permite visualizar un tránsito entre dos espacios de acción: un espacio interpersonal y de resistencia, y un espacio de integración funcional marcado por el mundo del trabajo. Esta separación no parece generar conflicto para los jóvenes, más bien el primero conformaría el espacio de reserva para poder funcionar en el segundo. De la misma manera que los aprendizajes realizados en el mundo del trabajo se capitalizan en las negociaciones en el campo del ocio.

Muchos de los jóvenes de hoy participan en agrupaciones y colectivos juveniles que no necesariamente responden a orgánicas reconocibles. Viven el día, pues suelen manifestar algunas esperanzas en el futuro, aunque ello pueda significar que oculten sus aspiraciones, si no logran verbalizarlas o si temen que sus sueños se transformen en las pesadillas de sus padres. Tienen muchas cosas que hacer como jóvenes; a veces esto que hacen les ocupa todo el tiempo libre, otras veces se convierte en una actividad más a la que le prestan algo de atención. Aun así, esto es un estilo de vida y las cosas que hacen delinean el trayecto de cómo se es joven en la época que les ha correspondido vivir.

Les convence la idea que la sociedad en que vivimos no está bien, creen que el gobierno del país no les toma en cuenta, les ve como chicos y chicas que no reflexionan sobre lo que les sucede; no es común que se les involucre en las decisiones sobre la sociedad que deseamos.

Las políticas sectoriales dirigidas a la juventud responden muy esporádicamente a sus necesidades, mientras que para la sociedad civil en general, los jóvenes buscan siempre su bienestar egoísta y son vulnerables y enfermos de juventud. Por lo tanto, sus demandas son una evidencia de aquel estado de euforia y locura propios de la edad.

Incluso así, persisten en agruparse, no con afán derrotista, sino ocupándose en sus intereses, y se juntan con otra gente a compartirlos. Si quieren organizar algo más grande y pueden adecuar sus objetivos a los que busca alguna institución pública que otorgue financiamiento, lo hacen sin dilemas. Saben hasta dónde pueden dar las instituciones del Estado y no les piden mucho más. Muchos de ellos se adecúan. Su meta es hacer lo que les gusta. Las opciones políticas generalmente los dividen, mientras que la cultura los relaciona. 
Otros se han dado cuenta que pueden aprovechar las circunstancias si se organizan bien, sólo así pueden expresar sus demandas al gobierno y a la sociedad. Saben que sus demandas son legítimas porque han recibido el apoyo de los adultos; sin embargo, este apoyo después se les quita y es algo muy desconcertante. De todas maneras, saben que si pudieron movilizarse una vez, lo podrán hacer de nuevo, aunque no ahora. Critican mucho al gobierno por burocrático. Se dan cuenta que los políticos se han aprovechado de sus demandas. Los tiempos de los adultos no coinciden con los suyos.

\section{b) La política de juventud y el diálogo con actores}

La política de juventud en Chile señala permanentemente su propio fracaso. Con excepción de aquellas experiencias locales que efectivamente entienden su trabajo bajo el concepto de joven como sujeto de derechos y no como beneficiario de políticas, el contenido de la política de juventud es un síntoma de la permanente invisibilización de la demanda de agrupaciones y colectivos juveniles, así como de la ausencia reiterada de espacios para la co-construcción de políticas.

Entre los contenidos más críticos de la política de juventud está la individualización, mas no una personalización, del vínculo con el poder político. Se ha encontrado que, entre los jóvenes y otros actores vinculados a juventud, la forma más recurrente de encontrarse es individual, con una mediación fuertemente burocratizada o totalmente despersonalizada, sin posibilidades de diálogo ni discusión.

¿Cuáles son los efectos sociales de una política de juventud con estas características? El primero, agrupaciones juveniles fuertemente atomizadas, que se desconocen entre sí y compitiendo por los mismos beneficios y recursos. Pero al mismo tiempo, jóvenes pragmáticos que generan nuevas formas de resistencia, nuevas condiciones de uso, de movilidad, de posibilidad y de proyección. Acciones juveniles en las que el desencanto no explica los espacios de interacción que las agrupaciones y colectivos últimamente están generando.

Por lo tanto, la acción a emprender debiera contener la apertura del debate entre los jóvenes, de forma que éstos puedan - sin la presión de un aparato organizativo o corporativo y desde la multiplicidad de roles que definen su incorporación como sujetos sociales- hacer visibles aquellas áreas en las que requieren del impulso de una política social y no compensatoria, que les permita acceder a la sociedad en mejores condiciones para enfrentar la vida. 
El objetivo que proponemos deriva de una premisa tácita. Para que haya una integración efectiva de la demanda juvenil a la política pública, debieran existir jóvenes capaces de ejercer ciudadanía plena; y éste es el gran desafío, que el contexto social no permite ni asegura todavía el respeto por los derechos de los jóvenes. Vivimos en un contexto social frágil que implica serias exclusiones de los grupos más desfavorecidos de la política, entre ellos, los jóvenes del país.

Creemos que la política de juventud ha de legitimarse como el instrumento cartográfico preciso para tocar «la diversidad de los universos juveniles [recogiendo] de una manera respetuosa, la capacidad de los propios jóvenes de representar sus demandas». Muy posiblemente su intervención no transoformará radicalmente los mapas cognitivos que se han elaborado hasta hoy, por los desafíos que presenta la política democrática hoy en día (sistemáticamente despojada de expectativas de mejoramiento a mediano y largo plazo y obligada a obtener resultados inmediatos), pero sí ha de poder incluir complejidad positiva en la relación de los jóvenes organizados con el poder público y las políticas sectoriales, la sociedad civil y los medios de comunicación.

\section{c) La demanda juvenil atrapada y cautiva}

Las agrupaciones y colectivos de jóvenes perciben que no hay mucho que hacer en el campo político; pero, si ahí ven algo que pueda servirles en su accionar como organización, investigarán los canales y gestionarán los recursos necesarios para conseguirlo. Lo anterior tiene un previsible efecto social, que es que la demanda de ciertas agrupaciones se estructura a partir de aquello que la política pública puede ofrecerles. Constituyen demandas que cautivan al poder público, pues se adecúan fácilmente a los objetivos que se proponen. Y, con ello, se convierten en demandas atrapadas en la oferta.

Las demandas cautivas son demandas concretas y factibles, funcionales a las políticas de gestión que construyen y organizan priorizando para cada actividad un espacio. Las agrupaciones y colectivos responden a la percepción que su accionar es problemático y, por tanto, se constituyen en prioridad para la política local. Los jóvenes que emprenden este tipo de demandas constituyen el correlato visible entre los discursos de identidad presentes en la acción juvenil de proyecto, y el discurso prefigurado por el poder político del joven de hoy.

Los jóvenes han desarrollado en el camino estrategias particulares para enfrentar el mundo que se les presenta. Algunos de sus itinerarios 
parecen responder a los estereotipos elaborados desde el poder político, la sociedad civil y los medios de comunicación; tanto es así que muchas acciones juveniles responden reflejando en un grado importante dichas miradas.

Estos mismos jóvenes tienen hoy otras vivencias, lo que los lleva también a abrir nuevos espacios de participación en el mundo que viven y, como consecuencia, a agruparse bajo otros estandartes. Fuertemente conectados con la industria cultural, la juventud de hoy imprime en sus itinerarios otra ética, estética y política, aunque parece que los conflictos que viven no son muy diferentes que los vividos por generaciones previas, aunque posiblemente éstos sean más radicales.

La reflexión toma sentido como problema cuando la intención es imaginar caminos donde coincidan los mapas cognitivos de los distintos actores con los itinerarios juveniles, y construir así una política pública adecuada a las vivencias y tránsitos del joven de hoy.

Es preciso una política de juventud que responda a las necesidades, intereses y, sobre todo, a los tiempos juveniles. Una cosa son los mapas que se elaboran, otra son los itinerarios juveniles, la política de juventud debiera ser un tercer elemento, actuar como mediador, un instrumento que ayude a construir un mapa lo más fidedigno posible de la geografía, y que ayude a no naufragar en el intento.

VALPARAÍSO (CHILE), NOVIEMBRE 2009

RECIBIDO: NOVIEMBRE 2009

ACEPTADO: DICIEMBRE 2009 


\section{REFERENCIAS BIBLIOGRÁFICAS}

CEPAL y OIJ (2008): Juventud y cohesión social en iberoamérica. Un modelo para armar. Santiago: CEPAL y OIJ.

— - (2004): La juventud en iberoamérica. Tendencias y urgencias. Santiago: CEPAL y OIJ.

CHAVEZ, MARIANA (2005): «Juventud negada y negativizada: representaciones y formaciones discursivas vigentes en la Argentina contemporánea». Última Década $\mathrm{N}^{\circ} 23$. Valparaíso: Ediciones CIDPA.

IBASE e PÓLIS (2008): Juventude e integração sul-americana: caracterização de situações tipo e organizações juvenis. 6 demandas para a construção de uma agenda comum. Relatório sul-americano. Rio de Janeiro: IBASE.

IBASE/PÓLIS/CIDPA (2009): Sociedades sudamericanas: lo que dicen jóvenes y adultos sobre las juventudes. Valparaíso: IBASE, PÓLIS y Ediciones CIDPA.

- (2008): Ser joven en sudamérica. Diálogos para la construcción de la democracia regional. Valparaíso: IBASE, PÓLIS y Ediciones CIDPA.

INJUV (2007): V Encuesta nacional de juventud. Santiago: INJUV.

— (2006): Segundo informe nacional de juventud. Condiciones de vida y políticas públicas de juventud desde la transición al bicentenario. Santiago: INJUV.

OIT (2007): Trabalho decente e juventude na América Latina. Brasília: OIT.

RODRÍGUEZ, ERNESTO (2000): «Juventud y políticas públicas en América Latina: experiencias y desafíos desde la gestión institucional». Revista Última Década $\mathrm{N}^{\circ} 13$. Viña del Mar: Ediciones CIDPA.

RUA, MARIA DAS GRAÇAS (1998): «As políticas públicas e a juventude dos anos 90» In: Jovens acontecendo na trilha das políticas públicas. Brasília: CNPD.

SERNA, LESLIE (1998): «Globalización y participación juvenil: en búsqueda de elementos para la reflexión». Revista JOVENes $\mathrm{N}^{\circ} 5$. México: Instituto Mexicano de la Juventud.

VIVAR, JULIETA; JUAN ClaUdio SILVA y OSCAR DÁVILA (2008): El secuestro de la demanda que cautiva. Valparaíso: Ediciones CIDPA. 